\title{
Fenología y Fenometría de una Variedad y una Línea de Frijol (Phaseolus vulgaris) en la Zona Occidental de El Salvador'1
}

\author{
Rolando Ventura Elías ${ }^{2}$
}

\section{COMPENDIO}

El cultivo del frijol, tiene factores que limitan su producción entre los cuales el comportamiento de los elementos atmosféricos es decisivo. El presente trabajo fue realizado durante los años de 1987,1988 y 1989 , en la zona Occidental de El Salvador, siembra de segunda, usando las variedades Rojo de Seda y APN 83; tuvo como objetivos, determinar la influencia del tiempo atmosférico en el desarrollo del cultivo y determinar las etapas fenológicas que sirvan de base en la planificación y ejecución de defensa y combate integral del cultivo.

Las etapas fenológicas que se establecieron fueron: emergencia, inicio de formación de guías, floración, formación de vainas, llenado de vainas y maduración. Las unidades de calor necesarias por cada etapa fenológica respectivamente resultaron ser: 12.89, 61.09, 89.08, 99.31, 114.97, 157.74 (temperatura base $18.9^{\circ} \mathrm{C}$ ). Los mismos fenómenos expresados en días después de la siembra corresponden a los promedios: $4.5,18.3,28.7,31.8,37.3$ y 54.2 respectivamente.

El elemento atmosférico de mayor influencia en la producción y desarrollo del cultivo es la precipitación pluvial, principalmente su Frecuencia, distribución y no cantidad. Similar efecto adverso, son los períodos clasificados microclimáticamente como sub-húmedo caliente.

Palabras claves adicionales: caracterización.

\section{INTRODUCCION}

El cultivo del frijol (Phaseolus vulgaris) constituye la principal fuente de alimento en la dieta de la población salvadoreña. Como todos los cultivos, el frijol tiene factores que limitan su producción entre los cuales los elementos atmosféricos son los más importantes.

Dada la importancia del frijol se realizó el presente estudio que tuvo como objetivos: determinar la influencia del tiempo atmosférico en el desarrollo del cultivo y establecer las etapas fenológicas de las variedades: Rojo de Seda y APN 83.

\section{REVISION DE LITERATURA}

Cardona et al. (1982), mencionan que varios factores ambientales pueden afectar la planta del frijol durante su desarrollo, Una exposición prolongada al exceso de humedad del suelo o al agua superficial, puede inducir clorosis en la

\begin{abstract}
$^{3}$
The common bean crop has limiting factors associated with production among which the behavior of atmospheric elements are decisive. This work was conducted from 1987 to 1989 in western El Salvador. Bean varieties 'Rojo de Seda' and APN 83 were planted during in the second cycle (sept-december). The objective of this work was to determine the influence of atmospheric conditions on the development of the bean plant and to determine the phonologic stages which serve as a base in the planning and execution of defense mechanisms for integrated management 01 " this crop.
\end{abstract}

The phonologic stages which were established were emergence, initial formation of branching, flowering, pod formation, pod filling and maturity. The necessary heat units for each phonological stage were: 12.89 , $61.09,89.08,99.31,114.97$, and 157.74 , respectively (base temperature of $18.9^{\circ} \mathrm{C}$ ). The same variables expressed in days after planting had the Following values: $4.5,18.3,28.7,31.8,37.3$ and 54.2 respectively.

Precipitation was the atmospheric clement which influenced production and development of the crop the most The Frequency and distribution were more important than the total amount. Similar adverse effects are also created by warm sub humid tropical climates.

planta y deficiencia de zinc, así mismo. afirman que el calor extremo, las heladas y la carencia de humedad ocasionan el marchitamiento de la planta, el cbamuscamiento de las hojas y aún la muerte de la planta, los vientos fuertes, el granizo y las partículas del suelo que el viento eleva pueden romper y raspar el tejido de las plantas.

Tapia y Camacho (1988) determinaron que la duración de las plantas de las distintas etapas está determinada por el hábito de crecimiento (Tipo I, II, III y IV); el clima (temperatura, fotoperíodo); el suelo (fertilidad, condiciones físicas) y el genotipo. La luz es otro factor que tiene un efecto directo en las etapas de desarrollo y la morfología de la planta. La fotosíntesis depende directamente de la luz; en sistemas de producción en asocio, por ejemplo maíz-frijol, el frijol

\footnotetext{
${ }^{1}$ Trabajo presentado en la XXXVI Reunión Anual del PCCMCA. San Salvador, El Salvador. marzo 1990.

2 Técnico en Agrometerología. CENTA, San Andrés, enero 1990. El Salvador, C.A.

${ }^{3}$ El abstraet es traducdón del compendio.

Publicado en Agronomía Mesoamericana. Vol. 2 (1991).
} 
compite por la luz. En tales condiciones se modifica su arquitectura y la producción de materia seca disminuye. graduados en mm, según normas OMM (Organización Meteorológica Mundial).

White (1985), establece que el frijol requiere de días cortos para que florezca. Los días largos demoran la floración y la maduración de la cosecha. Aunque existe mucha variabilidad en cuanto a la reacción varietaI de frijol al fotoperíodo, el efecto de cada hora adicional de la luz retarda la maduración de la semilla, que va de 2 a 6 días. El agua es el factor externo que determina más decisivamente el desarrollo del frijol común. El agua es reactivo de la fotosíntesis y elemento estructural, medio de transporte y regulador de la temperatura. Para la mayoría de los cultivos extensivos, las fases más críticas son el establecimiento o germinación y los cambios del estado vegetativo al reproductivo, incluyendo la floración y formación de cosecha (Jiménez, 1988).

\section{MATERIALES Y MÉTODOS}

Durante 1987, se ubicó en Cantón Joya del Zapote, Turín, Ahuachapán, a una elevación de 615 msnm. En 1988, en Cantón Salitrero, de Atiquizaya, Ahuachapán a $630 \mathrm{msnm}$. En 1989, en Cantón Izcaquilío, de Atiquizaya, Ahuachapán a $640 \mathrm{msnm}$. Los tres períodos, en siembra de segunda y relevó a maíz, con distanciamiento entre postura de 0.90 x $0.25 \mathrm{~m}$.

El trabajo se dividió en tres áreas, la primera enfocada a ciclo de vida del cultivo, que comprende fenología y fenometría, la segunda al comportamiento del tiempo atmosférico en la zona durante el ciclo del cultivo y la tercera a los requerimientos de grados de desarrollo para cada etapa fenológica de las variedades en estudio.

\section{A. Ciclo de vida del cultivo}

1 Fenología: Fue determinada haciendo un muestreo mixto (azar, sistemático), con frecuencia cada 2 días, observando el inicio de los fenómenos siguientes:

Fase vegetativa-reproductiva, emergencia, formación de guías, floración, formación de vainas (chiles), llenado de vainas y maduración.

2 Fenometría: Muestreo al azar, con frecuencia de 3 por semana durante las cuales se midió y cuantificó las estructuras siguientes: altura a partir del nudo cotiledonal, hojas verdes existentes, guías secundarias, flores, vainas llenas y vainas maduras.

\section{B. Comportamiento del tiempo atmosférico}

\section{Mesoclima}

a) Precipitación pluvial. Se usaron pluviómetros gra- duados en mm, según normas OMM (Organización Meteorológica Mundial).

\section{Microclima}

Se instaló micro-abrigo de $0.45 \mathrm{~m}$ de altura con aparatos de registro semanal.

a) Temperatura

Cálculos decádico (períodos de 10 años) de valores promedios.

b) Humedad Relativa

Cálculos decádicos de valores promedios.

\section{Comportamiento General Micro climático}

Se utilizó la clasificación microclimática desarrollada por Ventura y Zirnmerman (1986), que utiliza como límite térmico el valor de $25^{\circ} \mathrm{C}$ y como límite hídrico el valor de $70 \%$ de humedad relativa.

4. Requerimiento de grados de desarrollo diario o unidades de calor: González (1985) propuso la fórmula usada por Cooperative Extension Division of Agricultural Sciences, Universidad de California desde 1984, que se define de la siguiente manera:

$$
\begin{aligned}
& \mathrm{GDD}=\mathrm{K} \frac{(\mathrm{MAX}-\mathrm{T})^{2}}{\mathrm{AT}} \\
& \text { Donde: } \\
& \text { GDD }=\text { Grados de desarrollo diario } \\
& \mathrm{K}=0.25 \\
& \text { MAX = Temperatura máxima diaria } \\
& \mathrm{T}=\text { Temperatura Base (Temperatura mínima ab- } \\
& \text { AT }=\text { Rango diario }
\end{aligned}
$$

\section{Relación ETM/Precipitación Pluvial}

La demanda de agua del cultivo (ETM) definida por

$$
\begin{aligned}
\mathrm{ETM}= & \mathrm{ETP} \times \mathrm{Kc} \\
\mathrm{Kc}= & \begin{array}{l}
\text { Establecido por Doorembos y Kassam }(1979) \\
\text { citado por Jiménez } 1988 .
\end{array} \\
\mathrm{ETP}= & \begin{array}{l}
\text { Según Hargreaves }(\mathrm{ETP}=0.0075 \times \mathrm{RSM} \mathrm{X} \\
\mathrm{TMF})
\end{array}
\end{aligned}
$$




\section{RESULTADOS Y DISCUSION}

\section{Precipitación Pluvial}

El comportamiento de los elementos atmosféricos influye decididamente en el cultivo del frijol de los cuales los más determinantes son las condiciones pluviales pero sobre todo su distribución, seguido por el régimen técnico que a nivel microclimático provoca problemas fisiológicos traducido en diferencias fenológicas, longitud de períodos y efecto sobre la producción.

El presente estudio refleja que la fenología del cultivo expresa diferencias entre un período y otro, alargándose el ciclo (Cuadro 1 y 3) directamente proporcional a una buena distribución de la precipitación pluvial, principalmente a partir del fenómeno de inicio de formación de guías, hasta cierto límite.

La ausencia de precipitación o canícula, como una mala distribución que conlleva a deficiencias en el período de mayor demanda, provoca caída de estructuras fructíferas que impide la secuencia fenológica de la fase reproductiva. De forma similar se muestra que APN 83 es más sensible a períodos caniculares (Cuadros 1, 2 y 3). Las variedades en estudio requieren como mínimo la cantidad de $300.0 \mathrm{~mm}$.

Cuadro 1 Fenología del cultivo del frijol, siembra de segunda, variedades Rojo deSeda y APN 83, expresado en díassiembra, Ahuachapán. El Salvador. 1987 - 1989

\begin{tabular}{|c|c|c|c|c|c|c|}
\hline \multirow[t]{2}{*}{ Fenómeno } & \multicolumn{2}{|c|}{1987} & \multicolumn{2}{|c|}{1988} & \multicolumn{2}{|c|}{1989} \\
\hline & R.deS. & APN 83 & R.deS. & APN 83 & R.deS. & APN 83 \\
\hline Emergencia & 5 & 4 & 5 & 5 & 4 & 4 \\
\hline Formación de guías & 17 & 15 & 19 & 18 & 22 & 19 \\
\hline Floración & 25 & 28 & 29 & 28 & 31 & 31 \\
\hline Formación de vainas & 31 & 30 & 31 & 31 & 34 & 34 \\
\hline Llenado de vainas & 34 & 38 & 37 & 41 & 37 & 37 \\
\hline Maduración & 56 & $(*)$ & 54 & 59 & 50 & 52 \\
\hline
\end{tabular}

(*) No se registró (Influencia de canícula).
Relación ETM y Precipitación Pluvial

La ETM en función de temperatura, humedad relativa, y desarrollo del cultivo, durante los períodos de estudio, refleja la necesidad de demanda de agua del cultivo, éstos comparados con la precipitación pluvial demuestra la variable del comportamiento del clima (Cuadro 8), condición confirmada en Cuadro 6, así como la influencia en la fenología y fenometría (Cuadros 1,2), consecuentemente en la producción.

\section{Temperatura}

Los tres períodos de estudio registran diferencias de rango de temperatura (Cuadro 4). El ciclo del cultivo dividido en tres etapas: establecimiento del cultivo, fructificación y maduración. De registrarse en la segunda un mayor rango de temperatura que en los extremos, complementado con adecuada humedad, favorece la producción.

\section{Humedad Relativa}

El Cuadro 5 muestra las diferencias significativas en los tres períodos de estudio, producto en parte del comportamiento de la precipitación pluvial (Cuadro 3) consecuentemente su influencia en el desarrollo del cultivo (Cuadros 1 y 2).

\section{Comportamiento General Microclimático}

Un análisis simultáneo de los elementos temperatura y humedad relativa permite un mayor marco de referencia de comportamiento (Cuadro 6). La clasificación microclimática de los tres años refleja diferencias significativas.

El período crítico, 30- 50 D DS, para 1987 fue clasificado como sub-húmedo, en $100 \%$, para 1988 en $50 \%$ y para 1989 el $0.0 \%$. La clasificación refleja lo adverso y favorable a que se sometió las variedades, fenómeno confirmado por lo temprano y tardío de la fenología y la menor y mayor cantidad de estructuras productivas y vegetativas (Cuadros 1 y 2).

Cuadro 2 Edad en días después de siembra (DDS) de los máximos valores de estructuras productivas y vegetativas del cultivo del frijol siembra de segunda variedades Rojo de Seda y APN 83, Ahuachapán, El Salvador. 1987 - 1989.

\begin{tabular}{|c|c|c|c|c|c|c|c|c|c|c|c|c|}
\hline \multirow{3}{*}{ Fenómeno } & \multicolumn{4}{|c|}{1987} & \multicolumn{4}{|c|}{1988} & \multicolumn{4}{|c|}{1990} \\
\hline & \multicolumn{2}{|c|}{ Rojo de Seda } & \multicolumn{2}{|c|}{ APN 83} & \multicolumn{2}{|c|}{ Rojo de Seda } & \multicolumn{2}{|c|}{ APN 83} & \multicolumn{2}{|c|}{ Rojo de Seda } & \multicolumn{2}{|c|}{ APN 83} \\
\hline & MAX & DDS & MAX & DDS & MAX & DDS & MAX & DDS & MAX & DDS & MAX & DDS \\
\hline Altura & 77.9 & 42 & 58.3 & 35 & 90.8 & 49 & 72.8 & 49 & 140.0 & 49 & 118.3 & 55 \\
\hline Hoja & 17.9 & 51 & 17 & 43 & 20.1 & 49 & 22.0 & 41 & 31.2 & 46 & 39.0 & 55 \\
\hline Guías Secundarias & 3.0 & 42 & 16 & 35 & 3.2 & 30 & 3.6 & 41 & 3.3 & 34 & 5.0 & 37 \\
\hline Fiores & 2.8 & 41 & 3.5 & 35 & 4.1 & 32 & 4.3 & 31 & 7.6 & 49 & 9.4 & 43 \\
\hline Vainas en Formación & 12.1 & 44 & 6.3 & 39 & 13.7 & 45 & 12.9 & 42 & 13.9 & 49 & 13.3 & 52 \\
\hline Vainas & 6.5 & 47 & 4.9 & 41 & 12.5 & 54 & 11.8 & 51 & 19.6 & 55 & 21.2 & 61 \\
\hline
\end{tabular}


Cuadro 3 Precipitación decádica, durante el ciclo del cultivo del frijol Ahuachapán, El Salvador, 1987 -1989.

\begin{tabular}{|c|c|c|c|c|c|c|c|c|}
\hline \multirow[b]{2}{*}{ Mes } & \multirow[b]{2}{*}{ Década } & \multirow[b]{2}{*}{1987} & \multirow[b]{2}{*}{1988} & \multirow[b]{2}{*}{1989} & \multirow{2}{*}{$\begin{array}{l}\text { Valor * } \\
\text { Esperado }\end{array}$} & \multicolumn{3}{|c|}{$\begin{array}{c}\text { Diferencias } \\
\text { (Real - Esperado) }\end{array}$} \\
\hline & & & & & & 1987 & 1988 & 1989 \\
\hline \multirow[t]{3}{*}{ Septiembre } & $\begin{array}{ll}1 & 1\end{array}$ & 119.6 & 36.4 & 1.2 & & & & \\
\hline & 2 & 90.5 & 94.8 & 53.0 & & & & \\
\hline & 3 & 171.2 & 143.6 & 92.7 & & & & \\
\hline \multicolumn{2}{|c|}{ Total } & 381.3 & 274.8 & 146.9 & 375.0 & +6.3 & -100.2 & -228.1 \\
\hline \multirow[t]{3}{*}{ Octubre } & 1 & 3.1 & 23.8 & 88.4 & & & & \\
\hline & 2 & 17.5 & 0.0 & 114.1 & & & & \\
\hline & 3 & 0.0 & 16.8 & 0.0 & & & & \\
\hline Total & & 20.6 & 40.6 & 202.5 & 196.0 & -175.4 & -155.4 & +6.5 \\
\hline \multirow[t]{3}{*}{ Noviembre } & 1 & 0.0 & 0.0 & 53.8 & & & & \\
\hline & 2 & 0.0 & 0.2 & 19.3 & & & & \\
\hline & 3 & 0.0 & 0.0 & 0.0 & & & & \\
\hline Total & & 0.0 & 0.2 & 73.1 & 34.0 & -34.0 & -33.8 & +39.1 \\
\hline
\end{tabular}

(*) Cantidades normales de precipitación en mm. de los datos reales, Estación Ahuachapán, Almanaque Salvadoreño Servicio Meteorología e Hidrología. (registro 30 años).

Cuadro 4 Resumen decadico de valores extremos promedios de temperatura $\left({ }^{\circ} \mathrm{C}\right)$ en cultivo de frijol, siembra de segunda, variedades Rojo de Seda y APN 83 de Ahuachapán, El Salvador. 1987 -1989.

\begin{tabular}{|c|c|c|c|c|c|c|c|c|c|c|c|c|c|c|c|c|c|c|}
\hline \multirow[b]{3}{*}{ Decada } & \multicolumn{6}{|c|}{ Septiembre } & \multicolumn{6}{|c|}{ Octubre } & \multicolumn{6}{|c|}{ Noviembre } \\
\hline & \multicolumn{2}{|c|}{1987} & \multicolumn{2}{|c|}{1988} & \multicolumn{2}{|c|}{1989} & \multicolumn{2}{|c|}{1987} & \multicolumn{2}{|c|}{1988} & \multicolumn{2}{|c|}{1989} & \multicolumn{2}{|c|}{1987} & \multicolumn{2}{|c|}{1988} & \multicolumn{2}{|c|}{1989} \\
\hline & $\operatorname{Max}$ & Min & Max & Min & $\operatorname{Max}$ & Min & Max & Min & Max & Min & $\operatorname{Max}$ & Min & $\operatorname{Max}$ & Min & $\operatorname{Max}$ & Min & $\operatorname{Max}$ & Min \\
\hline 1 & 28.8 & 20.2 & 34.0 & 18.6 & 31.3 & 18.8 & 29.4 & 19.8 & 33.8 & 17.5 & 31.9 & 19.0 & 30.7 & 17.8 & 33.9 & 16.8 & 30.2 & 18.5 \\
\hline 2 & 30.5 & 19.5 & 33.0 & 18.5 & 34.2 & 19.6 & 31.0 & 18.0 & 33.1 & 17.7 & 30.8 & 19.0 & 31.0 & 19.8 & 33.9 & 17.0 & 30.2 & 18.5 \\
\hline 3 & 26.9 & 20.8 & 32.5 & 17.5 & 29.7 & 19.6 & 30.3 & 28.0 & 30.8 & 17.3 & 31.3 & 16.9 & & & & & & \\
\hline $\bar{X}$ Mes & 28.7 & 20.1 & 39.1 & 18.2 & 31.7 & 19.3 & 30.2 & 21.9 & 32.5 & 17.5 & 31.3 & 18.3 & 30.8 & 18.8 & 33.9 & 16.9 & 30.2 & 18.5 \\
\hline
\end{tabular}

Cuadro 6 Clasificación microclimática, registrado en cultivo de frijol, siembra de segunda Ahuachapán, El Salvador, 1987, $1988,1989$.

\begin{tabular}{|c|c|c|c|c|c|c|c|}
\hline \multirow[t]{2}{*}{ Mes } & \multirow[t]{2}{*}{ Década } & \multicolumn{2}{|l|}{1987} & \multicolumn{2}{|l|}{1988} & \multicolumn{2}{|l|}{1989} \\
\hline & & Clasificación & Símbolo & Clasificación & Símbolo & Clasificación & Símbolo \\
\hline & 1 & $\begin{array}{l}\text { Húmedo (1) } \\
\text { Semicaliente (1) }\end{array}$ & $\mathrm{H}_{1} \mathrm{SC}_{1}$ & $\begin{array}{l}\text { Húmedo (1) } \\
\text { Caliente (1) }\end{array}$ & $\mathrm{H}_{1} \mathrm{C}_{1}$ & $\begin{array}{l}\text { Subhúmedo (1) } \\
\text { Caliente (1) }\end{array}$ & $\mathrm{SH}_{1} \mathrm{C}_{1}$ \\
\hline \multirow[t]{3}{*}{ Septiembre. } & 2 & $\begin{array}{l}\text { Húmedo (1) } \\
\text { Semicaliente (1) }\end{array}$ & $\mathrm{H}_{1} \mathrm{SC}_{1}$ & $\begin{array}{l}\text { Húmedo (1) } \\
\text { Caliente (1) }\end{array}$ & $\mathrm{H}_{1} \mathrm{C}_{1}$ & $\begin{array}{l}\text { Subhúmedo (1) } \\
\text { Caliente (1) }\end{array}$ & $\mathrm{H}_{1} \mathrm{SC}_{1}$ \\
\hline & 3 & $\begin{array}{l}\text { Húmedo (1) } \\
\text { Semicaliente (1) }\end{array}$ & $\mathrm{H}_{1} \mathrm{SC}_{1}$ & $\begin{array}{l}\text { Húmedo (1) } \\
\text { Caliente (1) }\end{array}$ & $\mathrm{H}_{1} \mathrm{C}_{1}$ & $\begin{array}{l}\text { Húmedo } \\
\text { Semicaliente (1) }\end{array}$ & $\mathrm{H}_{1} \mathrm{SC}_{1}$ \\
\hline & 1 & $\begin{array}{l}\text { Subhúmedo (1) } \\
\text { Seimicaliente (1) }\end{array}$ & $\mathrm{SH}_{1} \mathrm{SC}_{1}$ & $\begin{array}{l}\text { Húmedo (1) } \\
\text { Caliente (1) }\end{array}$ & $\mathrm{H}_{1} \mathrm{C}_{1}$ & $\begin{array}{l}\text { Húmedo } \\
\text { Caliente (1) }\end{array}$ & $\mathrm{H}_{1} \mathrm{C}_{1}$ \\
\hline \multirow[t]{2}{*}{ Octubre } & 2 & $\begin{array}{l}\text { Subhúmedo (1) } \\
\text { Semicaliente (1) }\end{array}$ & $\mathrm{SH}_{1} \mathrm{SC}_{1}$ & $\begin{array}{l}\text { Subhúmedo (1) } \\
\text { Caliente (1) }\end{array}$ & $\mathrm{SH}_{1} \mathrm{C}_{1}$ & $\begin{array}{l}\text { Húmedo } \\
\text { Semicaliente (1) }\end{array}$ & $\mathrm{H}_{1} \mathrm{SC}_{1}$ \\
\hline & 3 & $\begin{array}{l}\text { Subhúmedo (1) } \\
\text { Caliente (2) }\end{array}$ & $\mathrm{SH}_{1} \mathrm{C}_{2}$ & $\begin{array}{l}\text { Húmedo (1) } \\
\text { Semicaliente (1) }\end{array}$ & $\mathrm{H}_{1} \mathrm{SC}_{1}$ & $\begin{array}{l}\text { Subhúmedo (1) } \\
\text { Semicaliente (1) }\end{array}$ & $\mathrm{SH}_{1} \mathrm{SC}_{1}$ \\
\hline Noviembre & 1 & $\begin{array}{l}\text { Subhúmedo (1) } \\
\text { Semicaliente (1) }\end{array}$ & $\mathrm{SH}_{1} \mathrm{SC}_{1}$ & $\begin{array}{l}\text { Húmedo (1) } \\
\text { Caliente (1) }\end{array}$ & $\mathrm{H}_{1} \mathrm{C}_{1}$ & $\begin{array}{l}\text { Subhúmedo (1) } \\
\text { Semicaliente (1) }\end{array}$ & $\mathrm{SH}_{1} \mathrm{SC}_{1}$ \\
\hline
\end{tabular}

(\%) en cultivo de frijol, siembra de segunda, variedades Rojo de Seda y APN 83. Ahuachapán, El Salvador. 1987 -1989.

\begin{tabular}{|c|c|c|c|c|c|c|c|c|c|}
\hline \multirow[b]{3}{*}{ Décadas } & \multicolumn{3}{|c|}{ Septiembre } & \multicolumn{3}{|c|}{ Octubre } & \multicolumn{3}{|c|}{ Noviembre } \\
\hline & 1987 & 1988 & 1989 & 1987 & 1988 & 1989 & 1987 & 1988 & 1989 \\
\hline & $\mathrm{X}^{*}$ & $\mathrm{X}$ & $\mathrm{X}$ & $\mathrm{X}$ & $X$ & $\mathrm{X}$ & $\mathrm{X}$ & $\mathrm{x}$ & $\mathrm{X}$ \\
\hline 1 & 74 & 78 & 68 & 61 & 72 & 72 & 57 & 70 & 72 \\
\hline 2 & 73 & 75 & 69 & 57 & 69 & 73 & 59 & 70 & 70 \\
\hline 3 & 77 & 73 & 77 & 54 & 75 & 67 & & & \\
\hline$\overline{\mathrm{X}}$ MES & 75 & 75 & 71 & 57 & 72 & 70 & 58 & 70 & 71 \\
\hline
\end{tabular}

* $\mathrm{X}=$ Promedio/Décadas 
Cuadro 7. Resumen promedio de requerimientos de grados de desarrollo o unidades de calor (Base $18{ }^{\circ} \mathrm{C}$ ) para cada etapa fenológica de las variedades de frijol Rojo de Seda y APN 83 Ahuachapán, El Salvador 1987-1989.

\begin{tabular}{lcc}
\hline FENOMENO & ROJO DE SEDA & APN 83 \\
\hline Emergencia & 12.89 & 12.89 \\
Formaclón de guías & 64.41 & 57.78 \\
Floración & 91.33 & 88.44 \\
Formación de vaina & 99.31 & 99.31 \\
Llenado de Vainas & 112.77 & 117.18 \\
Maduración & 153.24 & 162.25 \\
\hline
\end{tabular}

Requerimientos de grados de desarrollo o unidades de calor

Rojo de Seda más precoz que APN 83 (Cuadro 1) condición que se confirma al expresarse en grados de desarrollo o unidades de calor (Cuadro 7) registrándose diferencia del $5.5 \%$.

Al interpretar los fenómenos en forma aislada, durante las primeras tres etapas APN necesita menor cantidad de grados que Rojo de Seda, condición que se invierte en las dos etapas finales, aparentemente producto de las características genotípicas de cada variedad (Cuadro 7).

\section{CONCLUSIONES}

A Se determinaron seis etapas fenológicas del cultivo del frijol emergencia. inicio de formación de guías, floración formación de vainas, llenado de vainas y maduración.

B Prefloración, floración y formación de vainas son las etapas fenológicas más susceptibles a los períodos caniculares.

C Las unidades de calor necesarias para cada etapa fenológica fueron: $12.89,61.09,89.08,99.31,114.97,157.74$ (temperatura base $18.90{ }^{\circ} \mathrm{C}$ ) respectivamente.

D Frecuencia, distribución y no cantidad de precipitación pluvial, es el factor de mayor incidencia en la producción.

E Las clasificaciones de tiempo atmosférico, permite en parte, predecir las producciones para un área determinada.
Cuadro 8. Relación decádica, de precipitación pluvial y demanda de agua del cultivo de frijol (ETM) zona occidental de El Salvador, C.A. (1987. 1988)

\begin{tabular}{|c|c|c|c|c|c|c|c|}
\hline \multirow[b]{2}{*}{ Mes } & \multirow[b]{2}{*}{ Década } & \multicolumn{2}{|c|}{1987} & \multicolumn{2}{|c|}{1988} & \multicolumn{2}{|c|}{1989} \\
\hline & & ETM & $\mathbf{P P}$ & $\overline{\text { ETM }}$ & $\mathbf{P P}$ & ETM & $\mathbf{P P}$ \\
\hline & 1 & & 119.6 & 20.88 & 26.4 & 38.64 & 21.2 \\
\hline \multirow[t]{2}{*}{ Sept. } & 2 & 31. 77 & 90.5 & 32.76 & 94.8 & 53.01 & 53.0 \\
\hline & 3 & 45.98 & 171.2 & 37.60 & 143.6 & 57.73 & 92.7 \\
\hline \multirow{3}{*}{ Oct. } & 1 & 62.03 & 3.1 & 54.97 & 23.8 & 51.91 & 88.4 \\
\hline & 2 & 56.29 & 17.5 & 42.21 & 0.0 & 42.30 & 114.1 \\
\hline & 3 & 52.87 & 0.0 & 40.12 & 16.8 & 40.17 & 0.0 \\
\hline \multirow[b]{2}{*}{ Nov. } & 1 & 38.61 & 0.0 & 30.28 & 0.0 & 30.87 & 53.8 \\
\hline & 2 & 38.20 & 0.0 & & & & \\
\hline
\end{tabular}

\section{BIBLIOGRAFIA}

CARDONA, C.; F. C.A.; MORALES. F.J.; PASTOR CORRALES M. 1982. Problemas de campo en los cultivos de frijol en América Latina. Centro Internacional de Agricultura Tropical. Cali. Colombia. $100 \mathrm{p}$.

GONZALES. 1985 Formula sobre grados de desarrollo diario. Universidaá de Callifornia. Riverside 95502 USA. Cooperative Extension Division of Agricultural Sciences (Comunicación personal).

HARGREAVES, G.H. 1978. Clima y Agricultura. Comité Regional de Recurso. Hidraúlicos. Publicación No. 145. Tegucigalpa. Honduras. $20 \mathrm{p}$

JIMENEZ O., F. 1988. Necesidades hídricas de los cultivo. Centro Agronómico Tropical de Investigación y Enseñanza. Programa Manejo Integrado de Recursos Naturales. Turrialba. Costa Rica. $34 \mathrm{p}$.

TAPIA BARQUERO, H; CAMACHO HENRIQUEZ. A. 1988. Manejo Integrado de la Producción de Frijol basado en labranza cero. Deutsche Gesellschaft für Technische Zusammerlarbeit. Managua. Nicaragua. $181 \mathrm{p}$.

VENTURA ELIAS, R.; ZIMMERMANN MEJIA, R. 1986. Clasificación Microclimático del Cultivo del Algodonero. El Salvador. s.p.

WHITE, J. W. 1985. Conceptos básicos de Fisiología del frijol. Ing. López, M.I. Fernández., A Van Schoonhoven (ed). Frijol: Investigación y Producción. CIAT. Cali. Colombia, p. 43.60. 